\title{
Recovery of Unicellular Cyanobacteria from Nitrogen Chlorosis: A Model for Resuscitation of Dormant Bacteria
}

\author{
Niels Neumann Sofia Doello Karl Forchhammer \\ Interfaculty Institute of Microbiology and Infection Medicine Tübingen, Eberhard Karls University Tübingen, \\ Tübingen, Germany
}

\section{Keywords}

Metabolic quiescence $\cdot$ Cell cycle arrest · Glycogen

metabolism · ATP homeostasis · Starvation

\begin{abstract}
Nitrogen starvation induces developmental transitions in cyanobacteria. Whereas complex multicellular cyanobacteria of the order Nostocales can differentiate specialized cells that perform nitrogen fixation in the presence of oxygenic photosynthesis, non-diazotrophic unicellular strains, such as Synechococcus elongatus or Synechocystis PCC 6803, undergo a transition into a dormant non-growing state. Due to loss of pigments during this acclimation, the process is termed chlorosis. Cells maintain viability in this state for prolonged periods of time, until they encounter a useable nitrogen source, which triggers a highly coordinated awakening process, termed resuscitation. The minimal set of cellular activity that maintains the viability of cells during chlorosis and ensures efficient resuscitation represents the organism's equivalent of the BIOS, the basic input/output system of a computer, that helps "booting" the operation system after switching on. This review summarizes the recent research in the resuscitation of cyanobacteria, representing a powerful model for the awakening of dormant bacteria.
\end{abstract}

(C) 2021 The Author(s)

Published by S. Karger AG, Basel

\section{Introduction}

Nutrient starvation can be detrimental to organisms when it leads to metabolic disorder that impairs vital cellular processes. A successful strategy to survive such periods of nutrient deprivation that is particularly widespread in prokaryotes is the transition into a dormant state. In this state, the metabolic processes are reduced to an absolute minimum. The remaining activities just ensure survival and enable the dormant cells to awake and resume growth once the limiting nutrient becomes available again. It is assumed that in natural environments a majority of the bacteria reside in a dormant state, and these cells comprise a "seed bank" - a reservoir - that can be resuscitated when favourable conditions occur. This mechanism is largely responsible for the fitness of bacterial populations and contributes to the sudden appearance of bacteria in the environment and the spreading of antibiotic resistances and bacterial pathogens. An example of dormant bacteria acting as a reservoir of antibiotic resistance is presented in the study by Udikovic-Kolic et al. [2014], where manure supplementation of soil is shown to result in proliferation of diverse soil-resident bacteria, some carrying antibiotic resistance genes.

The ways in which the cells enter dormancy and recover from the dormant stage are rather diverse. Some bacteria form endospores or exospores, others create en- karger@karger.com www.karger.com/mip

Karger $\stackrel{\text { ' }}{5}$

GOPEN ACCESS
(C) 2021 The Author(s)

Published by S. Karger AG, Basel

This is an Open Access article licensed under the Creative Commons Attribution-NonCommercial-4.0 International License (CC BY-NC) (http://www.karger.com/Services/OpenAccessLicense), applicable to the online version of the article only. Usage and distribution for commercial purposes requires written permission.
Correspondence to:

Karl Forchhammer, karl.forchhammer@uni-tuebingen.de 
capsulated cysts or even generate apparently non-differentiated persister cells, which is the case in Mycobacterium tuberculosis [Keren et al., 2011]. Despite the differences among these quiescent states, a common requirement in this survival strategy is the ability to reactivate metabolism upon encountering permissive conditions.

Studying the molecular mechanisms supporting these cellular processes is complicated for several reasons. A bacterial population that has undergone the transition into a dormant state is usually highly heterogeneous, since phenotypical variation can be beneficial in the emergence from starvation-induced growth arrest [Moreno-Gámez et al., 2020]. In many cases, including populations of nutrient-starved Flavobacterium columnare and Brucella suis cells, only a small proportion of the cells remain viable [Arias et al., 2012; AlDahouk et al., 2013]; in others, such as the germination of akinetes of filamentous heterocyst-forming cyanobacteria, re-activation of metabolism is highly asynchronous [Perez et al., 2018]. This heterogeneity precludes the employment of powerful molecular methods that rely on homogenous populations, such as metabolomics, proteomic or transcriptomic studies. Therefore, in many cases, the behaviour of dormant cells can only be studied using single-cell analysis, which still has many limitations. One of the rare cases where transition into and out of the dormant state occurs in a population-wide coherent manner is the case of nitrogen starvation-induced chlorosis in some unicellular cyanobacterial strains, such as Synechococcus elongatus and Synechocystis PCC 6803. Following a brief description of nitrogen starvation-induced chlorosis, the present article provides a consolidated review of our current knowledge on the resuscitation of these cyanobacteria from prolonged nitrogen chlorosis.

\section{Entry into Chlorosis}

That nitrogen starvation leads to bleaching of cyanobacterial cultures was already described in 1910 and this process was termed "chlorosis" [Boresch, 1910]. Only about 70 years later, studies in S. elongatus showed that the rapid bleaching caused by nitrogen starvation, a colour change from deep blue-green to yellowish-greenish, was mainly caused by the degradation of the blue phycobiliproteins [Allen and Smith, 1969]. These proteins constitute the light-harvesting antenna system for the photosynthetic reaction centres, the phycobilisomes (PBS) [Grossman et al., 1993]. By screening for mutants with a non-bleaching phenotype on nutrient-deficient plates in the 1990s, $n b l$ (non-bleaching) genes were identified in the laboratory of A. Grossman [Collier and Grossman, 1994]. These $n b l$-genes are responsible for phycobiliprotein degradation upon various stress conditions, including nitrogen starvation or high-light stress. Until the mid1990s, it was assumed that the chlorotic cells lost viability, until a study by Görl et al. [1998] showed that chlorotic Synechococcus cells maintain viability for extended periods of time [reviewed in Schwarz and Forchhammer, 2005]. This study revealed a gradual decline in cellular activities following the initial phycobiliprotein degradation, until the cells entered a dormant-like state with almost no detectable metabolic activity. Based on these observations, the chlorosis process could be divided into 3 phases, with phase 1 being the rapid degradation of phycobiliprotein occurring within 1-2 days, followed by phase 2, the gradual decline of metabolic activity (concomitant with a decline in chlorophyll $a$ ) within the following 8-14 days, and finally, the terminal phase 3 , the dormant state with a low basal level of photosynthetic activity [Görl et al., 1998]. Concomitant with the gradual decline of metabolic activity, the cells undergo substantial intracellular re-organization through proteolysis, in particular with respect to the degradation of the intracellular membrane system, the thylakoids, which accommodate the molecular machines of the photosynthetic electron transport chain including photosystem I and II. However, a low level of photosynthetic activity involving PSI and PSII activity is preserved during prolonged chlorosis, which was shown to be required to maintain long-term cell viability [Sauer et al., 2001]. Since no net yield of photosynthesis could be determined, it was suggested that the photosynthetic electrons are recycled through a water-towater cycle, where oxygen, produced through water splitting at PSII, serves as electron acceptor for PSI-reduced ferredoxin [Sauer et al., 2001]. Considering the severe decomposition of intracellular structures after long-term chlorosis, it is astonishing that cells readily re-green within 2 days and resume growth following the addition of a nitrogen source [Görl et al., 1998; Sauer et al., 1999; Sauer et al., 2001; reviewed in Schwarz and Forchhammer, 2005].

Synechocystis sp. 6803 is a unicellular cyanobacterium only distantly related to $S$. elongatus [Robertson et al., 2001]. It is metabolically more versatile than S. elongatus as it is not strictly photoautotrophic and is able to synthesize various reserve polymers not present in S. elongatus, such as the nitrogen storage compound cyanophycin (CP) [Stephan et al., 2000] or the polymer polyhydroxybutyrate (PHB) [Hauf et al., 2013; reviewed in Koch et al., 2020]. Despite these differences, the basic nitrogen-star- 


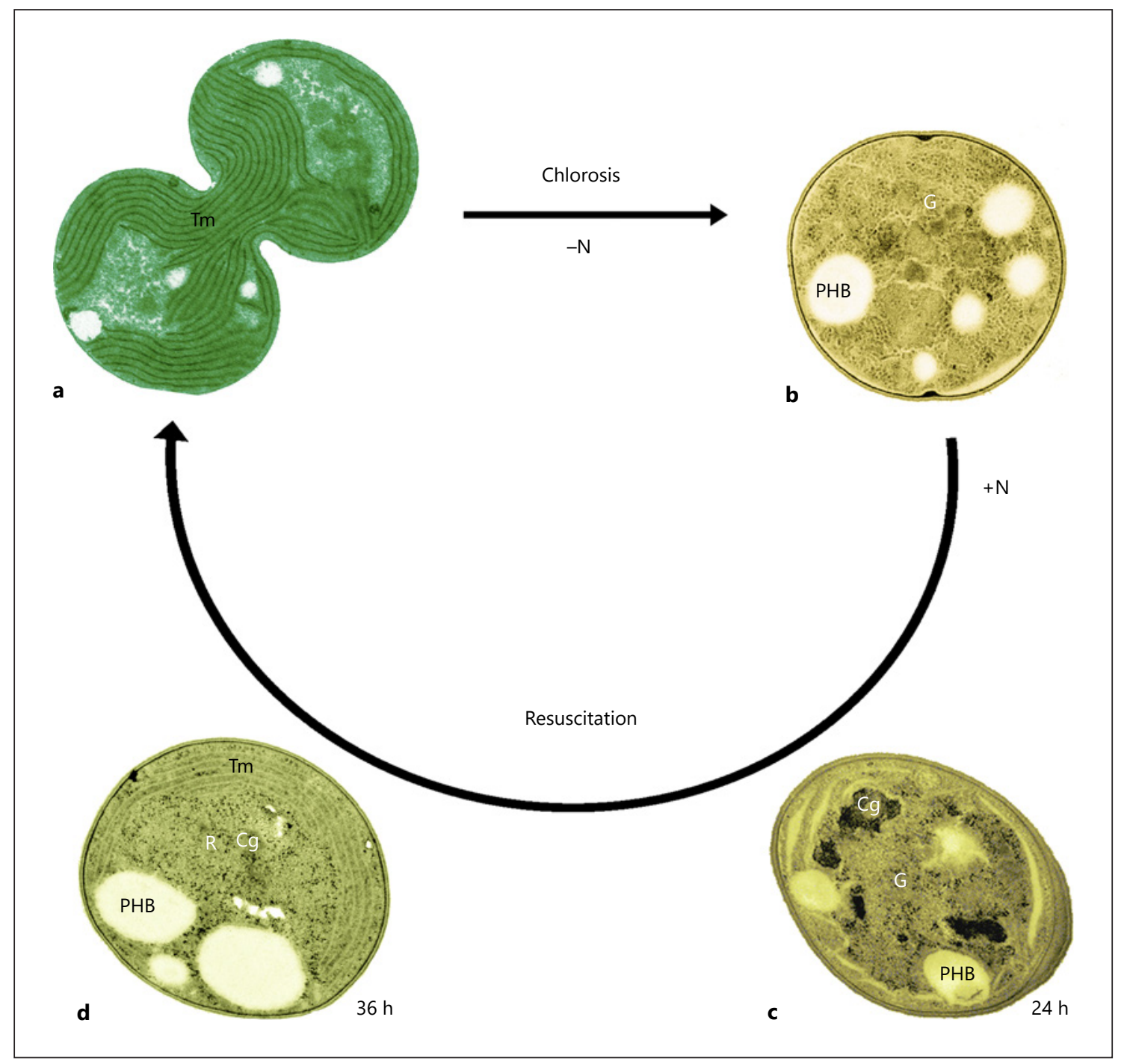

Fig. 1. Synechocystis cells during developmental transitions through nitrogen starvation and resuscitation. a Cell in exponential growth phase. b Chlorotic cell starved for 1 month. c Cells during the first $24 \mathrm{~h}$ of resuscitation. d Cells after 36 h of resuscitation. G, glycogen granules; PHB, polyhydroxybutyrate granules; $\mathrm{Cg}$, cyanophycin granules; Tm, thylakoid membrane; R, ribosomes.

vation acclimation response seems to be highly conserved. Similar to S. elongatus, Synechocystis employs a set of $n b l$ genes under the control of a conserved sensor kinase (Hik33/NblS) to degrade phycobiliproteins, which degrades the photosynthetic apparatus and tunes down metabolic activity until a dormant-like state is attained, from which it rapidly recovers after supplementation with combined nitrogen [reviewed in Schwarz and Forchhammer, 2005]. A distinguishing feature of the strains is the accumulation of large amounts of PHB in Synechocystis during prolonged chlorosis [Schlebusch and Forchhammer, 2010; Hauf et al., 2013; Damrow et al., 2016].
However, mutants defective in $\mathrm{PHB}$ synthesis are neither impaired in the chlorosis response, nor in the ability to recover from nitrogen starvation [Klotz et al., 2016; Koch et al., 2020], indicating that PHB metabolism is not directly involved in surviving nitrogen deprivation. Unlike $\mathrm{PHB}$, glycogen seems to be a major carbon polymer supporting nitrogen starvation; both Synechocystis and S. elongatus rapidly accumulate large amounts of this polymer upon nitrogen limitation or upon inhibition of glutamine synthetase (GS) activity [Klotz et al., 2015]. However, mutants that are defective in glycogen synthesis ( $g \lg C$ mutants or $g \lg A 1 / g \lg A 2$ double mutants) display 
metabolic deficiencies during nitrogen starvation and are impaired in phycobiliprotein degradation and, therefore, show a non-bleaching phenotype. After a few days under nitrogen-deficient medium, they lose viability [Gründel et al., 2012; Hickman et al., 2013]. The synthesis of glycogen is accompanied by the concomitant induction of expression of glycogen catabolic genes, for which the SigE and Rre37 regulators are responsible [Azuma et al., 2011; Osanai et al., 2014]. Although this appears paradoxical, it illustrates that a genetic program exists that "anticipates" the recovery from nitrogen starvation (see below).

\section{Resuscitation from Chlorosis: A Global Analysis}

A first global and comprehensive analysis of the resuscitation of chlorotic Synechocystis cells was performed by Klotz et al. [2016]. When fully developed, long-term chlorotic Synechocystis cells were supplied with nitrate, and it could be shown that they started to re-green and regain photosynthetic activity after about $24 \mathrm{~h}$. After about 2 days, photosynthetic performance was almost completely restored, and after 3 days, cell division was resumed. Figure 1 provides a graphical representation of these developmental transitions. At a first glance, the retarded appearance of photosynthetic pigments might give the impression that the cells only respond to the addition of a nitrogen source with a delay of approximately a day. However, examination at the molecular level reveals that the apparent 24-h lag phase is in fact a highly dynamic period. As shown by transcriptomic and proteomic analysis, the basic operating system of the cells, which during chlorosis has been turned down to a minimum, must be re-established before the cells are able to re-green and rebuild the complex intracellular machineries of photosynthesis [Klotz et al., 2016; Spat et al., 2018]. It transpired that the entire process follows a highly orchestrated genetically encoded program, evident at all levels of cellular activity.

At the metabolic level, resuscitation can be divided into two principal phases. In the first phase, immediately after nitrate addition, cells turn on a heterotrophic-like metabolism, based on glycogen consumption and oxygen-dependent respiration. In the second phase, after about $24 \mathrm{~h}$, when the photosystems reappear, cells switch to a mixotrophic metabolism, where glycogen utilization co-occurs with $\mathrm{CO}_{2}$ fixation and oxygenic photosynthesis. Only in the fully recovered state do they finally resume the classical photoautotrophic metabolism and return to vegetative growth. A key to the understanding of the resuscitation program is the underlying glycogen metabolism, which provides the cells with energy and metabolic precursors (see Fig. 2 for an overview of the principal pathways of glycogen metabolism in Synechocystis). Therefore, glycogen is pivotal to the viability of chlorotic cells. The Synechocystis genome harbours two paralogous genes for glycogen synthetases, glgA1 (sll0945) and $g l g A 2$ (sll1393). Knockout of only one of the two $g \lg A$ paralogues results in mutants that synthesize the same amount of glycogen as the wild type following nitrogen step down. Nevertheless, these two mutants display striking phenotypic differences upon nitrogen starvation. Whereas the glgA2 mutants are able to carry a wild-type response to chlorosis, the $g \lg A 1$ mutants as well as the $g \lg A 1 / \operatorname{glg} A 2$ double mutants show impaired chlorosis: instead of a step-wise degradation of the photosynthetic pigments, the mutants do not initially respond to nitrogen depletion, but after a while they turn white and lose viability [Koch et al., 2019]. This suggests that only glycogen produced by GlgA1 is relevant for survival. The molecular basis of this difference is unclear and is currently under investigation.

Similar non-redundant pairs of glycogen metabolic genes are found for the paralogues of glycogen phosphorylase, $g \lg P 1$ (sll1356) and $g l g P 2$ (slr1367), as well as for the paralogues for glycogen-debranching enzyme $g \operatorname{lgX} 1$ (slr0237) and $g \operatorname{lgX} 2$ (slr1857). The analysis of the respective mutants showed that only GlgP2 supports the resuscitation program. The $g \lg P 2$ mutants (still able to produce GlgP1), as well as the $g l g P 1 / g l g P 2$ double knockout mutants, are unable to be resuscitated from chlorosis, although the initial process of chlorosis proceeds normally, as deduced from the ordered degradation of pigments [Doello et al., 2018]. The poor resuscitation of the various $g l g A$ and $g l g P$ mutants is depicted in Figure 2b. The glycogen phosphorylase reaction releases Glc-1P, which is further converted by phosphoglucomutase (PGM, sll0726) to generate Glc-6P (see Fig. 2a). This metabolite can either enter the Embden-Meyerhof-Parnas pathway (EMP), or it can be oxidized by glucose-6P dehydrogenase (encoded by the $z w f$ gene) to 6P-gluconate, which is further metabolized by the oxidative pentose phosphate (OPP) cycle or the Entner-Doudoroff (ED) pathway. Whereas mutants with a deficient EMP pathway recover almost like the wild type from the chlorotic state, $z w f$ knockout mutants are strongly impaired in resuscitation. A mutant deficient in only the ED pathway is less severely affected than a mutant with an impairment in the OPP pathway, showing that the latter is the most important glycogen catabolic pathway in recovering chlorotic cells [Doello et al., 2018]. 


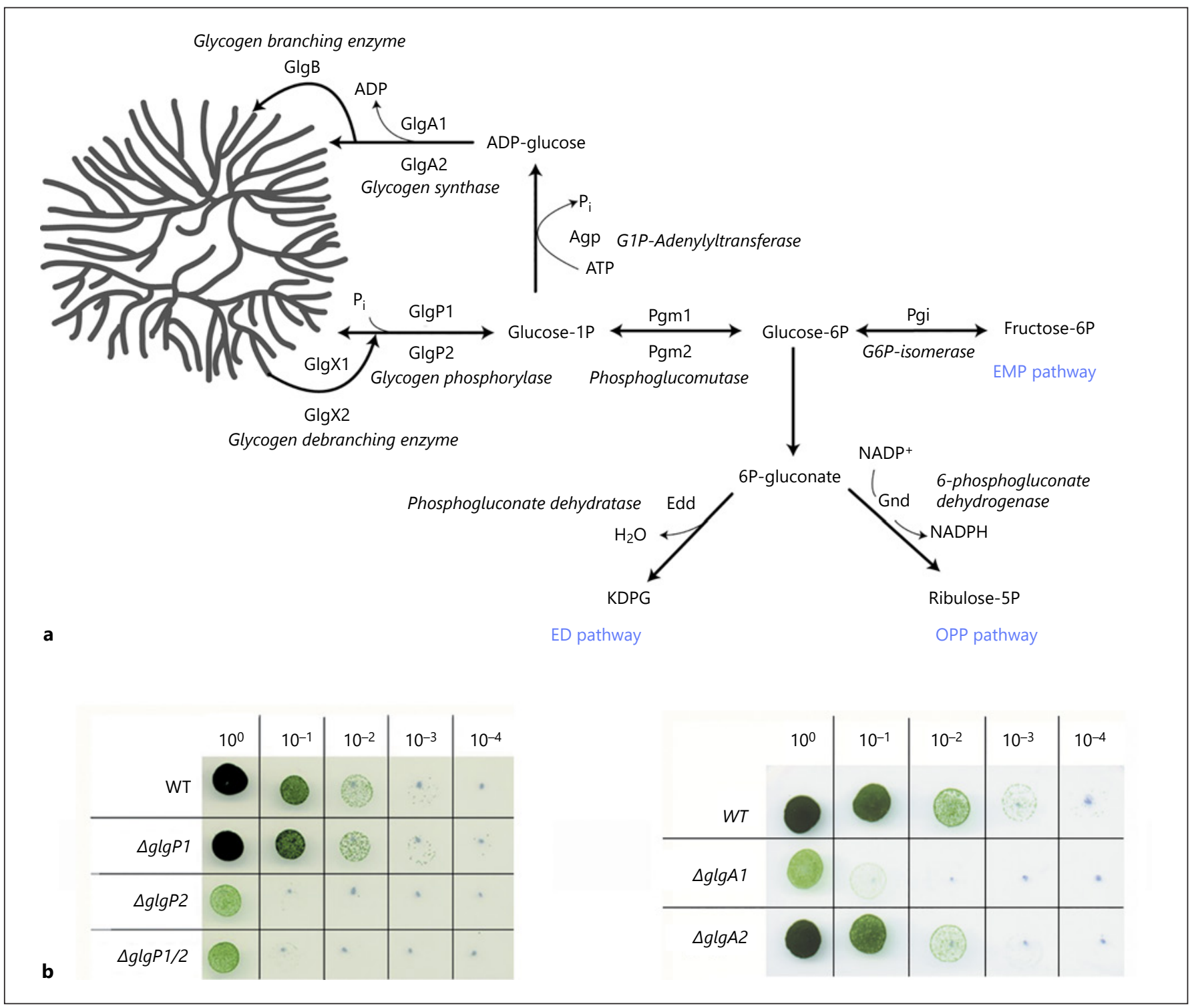

Fig. 2. Schematic representation of glycogen metabolism in Synechocystis and its involvement in chlorosis and resuscitation. a Depiction of reactions in glycogen metabolism and glycogen catabolic pathways in Synechocystis. b Resuscitation of chlorotic cells tested by drop plate assay. From a serial dilution of chlorotic cultures $\left(0-10^{-4}\right.$ dilution as indicated), $5 \mu \mathrm{L}$ were dropped on nitrate-supplied BG11 plates and incubated until colonies appeared. Resuscitation efficiency of wild type (WT) is compared to various mutants deficient in glycogen phosphorylase $(\triangle g \lg P 1, \Delta g \lg P 2, \Delta g \lg P 1 / 2)$ or glycogen synthase $(\triangle g \lg A 1, \Delta g \lg A 2)$.

When the cells started to respire and to degrade glycogen upon the addition of nitrate to chlorotic cultures, the low level of photosynthetic activity, which is still measurable in the chlorotic state, is completely shut down. Photosynthetic oxygen evolution only reappears at the end of the first phase of resuscitation, indicating a switch to a mixotrophic metabolism. During this period, which takes place about 1 day after addition of the nitrogen source, re-greening becomes visible, indicating the re-installation of the photosynthetic machinery. Electron micrographs reveal that in this second phase of resuscitation, the cells reconstruct the thylakoid system de novo (see Fig. 1d). After about 2 days, most of the cells have recovered their photosynthetic machineries and are able to switch metabolism toward vegetative growth. With the onset of cell division, 2-3 days after the addition of a ni- 
Fig. 3. Sodium-dependent bioenergetic processes in nitrogen-starved cells. Depiction of the components of the respiratory chain and the ATP synthesis machinery in the plasma membrane of Synechocystis.

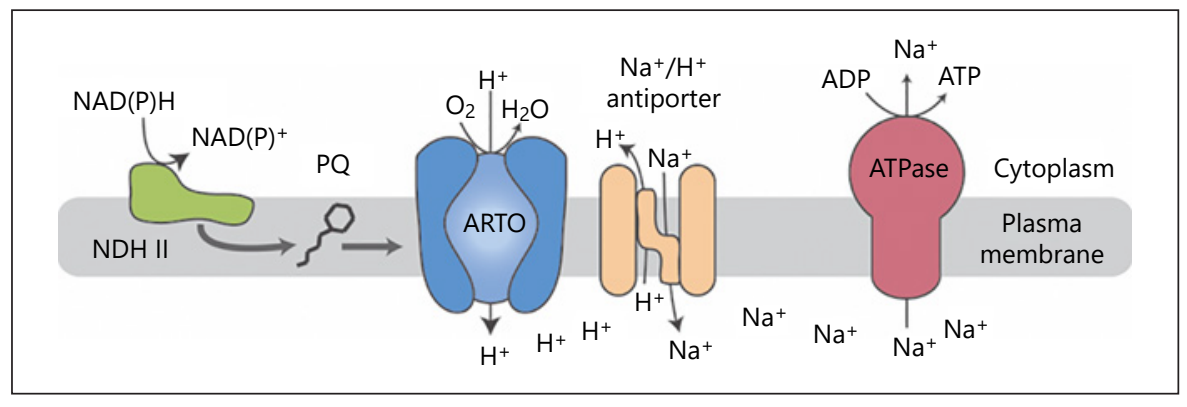

trogen source, the resuscitation program is complete, and the cells exhibit typical vegetative growth. During chlorosis, the cells apparently become arrested in a pre-divisional state; they contain on average double the amount of chromosomal DNA than actively growing cells. At the end of the resuscitation program, the average cell size, as well as the average chromosome copy number, decrease to half the values of chlorotic cells, which is indicative of ongoing cell division [Klotz et al., 2016].

\section{The Start of Resuscitation}

\section{Bioenergetics of Awakening}

During chlorosis, metabolic reactions are reduced to a minimum and the intracellular energy charge is kept low; the ATP content is maintained at a basal level, which constitutes the minimum to maintain cell survival. Thus, the ATP levels in nitrogen-starved cells are reduced by up to $25 \%$ of the value in vegetatively growing cells. Upon addition of a nitrogen source to chlorotic cells, the first detected cellular response is a rapid increase in the ATP content. However, the extent of this increase is dependent on the form in which nitrogen is provided. Addition of sodium nitrate to chlorotic cells triggers a $100 \%$ increase in the ATP content within 20 min, whereas supplementation with potassium nitrate leads to a slower increase in the ATP levels (approximately 20-30\% less compared to sodium nitrate). Doello et al. [2021] investigated this peculiar phenomenon and have discovered a so far unrecognized and unique bioenergetic strategy in nitrogenstarved cells.

During prolonged chlorosis, the thylakoid membranes, which accommodate the majority of the F-type ATPase complexes, are degraded. Consequently, chlorotic cells rely on the ATP synthases located in the plasma membrane, which are able to use a sodium motive force to power ATP synthesis. When resuscitation is started by the addition of sodium nitrate, the sudden rise of the extracellular sodium concentration leads to an increased so- dium motive force, which causes an instantaneous increase in the ATP levels. When potassium nitrate is used to induce resuscitation, the concentration of sodium remains constant, and the increase in the ATP content results from a metabolically regulated response to the availability of a nitrogen source.

When nitrogen assimilation initiates, the cellular ATP demand dramatically increases due to the high turnover rate of the energy-consuming GS reaction. A concomitant increase in ATP synthesis is, therefore, essential to ensure efficient nitrogen assimilation and to avoid drainage of the cellular ATP levels. How cells achieve this tight control of the ATP homeostasis has not yet been entirely deciphered; however, it has been shown that activation of the GS reaction is involved in this regulatory mechanism, because the increase in ATP levels in response to the availability of nitrogen is sensitive to MSX, a specific GS inhibitor. Additionally, upon addition of a nitrogen source, ATP synthesis requires glycogen degradation and respiration. This was demonstrated by the lack of an increase in the ATP content in a GlgP-negative mutant, which is impaired in glycogen catabolism, and in wildtype cells when they are treated with potassium cyanide $(\mathrm{KCN})$, an inhibitor of the respiration. Moreover, specific inhibition of electron transfer from the plastoquinone pool (PQ) to the cytochrome $b_{6} f$ complex, which is exclusively found in the thylakoid membranes, reveals that respiration takes place at the plasma membrane in chlorotic cells undergoing the early steps of resuscitation [Doello et al., 2021]. It is, therefore, tempting to speculate that nitrogen assimilation through the GS reaction initiates glycogen breakdown, thereby generating reducing equivalents, which then activate respiration at the cytoplasmic membrane. The plasma membrane is equipped with an alternative respiratory chain consisting of a type II NADH:quinone oxidoreductase (NDH II) and an alternative respiratory terminal oxidase (ARTO) [Baers et al., 2019]. In fact, we could show that NDH II is one of the most highly accumulated proteins in the chlorotic state 
[Spat et al., 2018]. In vegetative cells, this respiratory system is coupled to the generation of a sodium motive force to energize sodium-dependent bicarbonate uptake [Pils and Schmetterer, 2001]. In nitrogen-starved cells, where inorganic carbon fixation is arrested, this bioenergetic system ensures energy provision in the standby mode and to support the initial phase of resuscitation (Fig. 3).

\section{Resumption of Nitrogen Assimilation}

The activity of GS is subject to tight regulation. In cyanobacteria, GS activity is controlled by the inhibitory proteins IF7 and IF17, whose synthesis is under global nitrogen control and is strongly downregulated during nitrogen starvation [Bolay et al., 2018]. Moreover, the cells express a second, nitrogen starvation-specific GS gene, $g \ln N$ [Reyes and Florencio, 1994]. In S. elongatus, GlnN helps recovery of the cells from long-term nitrogen starvation [Sauer et al., 1999]. Thus, the cells maintain highly active nitrogen assimilation capacity during chlorosis. As soon as nitrogen becomes available again, GS starts working, thereby initiating the resuscitation program. Nitrogen assimilation then operates at full capacity, with the inhibitory peptides IF7 and IF187 only being induced toward the end of resuscitation [Spat et al., 2018]. In agreement with these findings is the observation that the noncoding regulatory RNA NsiR4, which prevents expression of IF7, remains highly expressed in the initial phase of resuscitation. The assimilation of combined nitrogen initially exceeds the pace of protein synthesis, since the entire translational machinery first has to be re-installed. The excess of assimilated nitrogen is transiently stored as CP [Watzer and Forchhammer, 2018], which is deposited as a granular structure in the cytoplasm (see Fig. 1c; structures indicated by $\mathrm{Cg}$ ). When the protein synthesis machinery is fully operational, the CP material is recycled for synthesis of cellular building blocks. This strategy enables cells to store any available nitrogen for subsequent utilization in an environment with fluctuating and transient nitrogen supply. Physiological comparison of mutants deficient in CP synthase (cphA) with wild-type cells demonstrated that in the absence of CP synthesis cells are unable to recover completely when nitrogen is only transiently available to the cells. By contrast, wild-type cells can resuscitate successfully and return to vegetative growth [Watzer and Forchhammer, 2018].

\section{Early Events in Transcriptional Regulation}

To reveal the molecular events occurring during the process of resuscitation, a detailed transcriptome study has been performed [Klotz et al., 2016]. To achieve this, a high-density microarray covering the entire chromosome and the 7 plasmids was used, probing all 8,916 transcripts of coding and non-coding RNA. RNA was extracted at 5 different time points (after 0, 4, 12, 24 and $48 \mathrm{~h}$ after nitrate addition), starting with fully developed chlorotic cells and finishing with completely re-greened cells. A total of 1,570 differentially regulated genes were detected, which corresponds to $17.6 \%$ of the entire transcriptome. Of these genes, approximately one-third comprises non-coding RNA. The changes in the transcriptome during the resuscitation process perfectly reflect the chronological order of cellular processes. The majority of the differentially regulated genes belong to the early responding genes, whose expression changes after about 4 h. They comprise 425 and 626 up- or downregulated genes, respectively, compared with the dormant state. The upregulated group of genes includes the entire set of genes encoding the translational apparatus, as well as metabolic genes, particularly those involved in nitrate/ nitrite assimilation, genes encoding enzymes for co-factor biosynthesis, ATP synthesis and $\mathrm{CO}_{2}$ fixation. The latter finding is striking, as it hints at a role for $\mathrm{CO}_{2}$ fixation prior to the onset of photosynthesis. In agreement with this suggestion, Doello et al. [2018] detected $\mathrm{CO}_{2}$ fixation activity as soon as $10 \mathrm{~h}$ after the initiation of resuscitation, well before the onset of oxygen evolution. Surprisingly, the genes encoding the enzymes required for glycogen catabolism are not strongly upregulated at the transcriptional level. In fact, the glycogen catabolic genes are already upregulated in response to the nitrogen step down, concomitant with the synthesis of glycogen. Proteome analyses confirm that the respective proteins are maintained at elevated levels throughout the chlorotic state. This demonstrates an "anticipatory principle" in the acclimation toward nitrogen starvation. The decisive catabolic enzymes are prepared in standby mode, awaiting the availability of nutrient. This necessarily implies that the enzymes, such as glycogen phosphorylase, have to be kept in an inactive state, in order to prevent premature glycogen consumption. Preliminary data indicate a key role for PGM in the control of glycogen breakdown [unpubl. data].

As deduced from the dynamics of the transcriptome, the early responses reflect the re-installation of the basic operation system of the cells, the core anabolic reactions, energy metabolism and the translational apparatus, enabling the cells to re-configure the complex cellular structures that were degraded during chlorosis. As deduced from this study, the transcriptional machinery itself, in particular the components of the RNA polymerase, does 
not change in expression as strongly as the translational machinery. The changes are more subtle and affect specific regulatory components. During nitrogen starvation, the transcriptional machinery switches from use of the house-keeping SigA-dependent RNA polymerase to use of alternative sigma factors, in particular, SigB, SigC and SigE [Imamura and Asayama, 2009; Heilmann et al., 2017]. In particular, SigC has been shown to promote late-stage gene expression and nitrogen promoter recognition [Asayama et al., 2004]. SigE mediates induced expression of various carbon catabolic genes during nitrogen starvation (see above). During resuscitation, however, the transcriptional machinery has to be re-wired toward SigA-dependent transcription, which is responsible for the expression of the core operating system of the cell. The extensive re-organization of the transcriptome agrees with a dominant role for different sigma factors that affect global transcriptional patterns [Srivastava et al., 2020]. The small non-coding 6S RNA may have a key role in sigma factor transition. The 6S RNA is widely distributed in prokaryotes and acts as a global regulatory RNA involved in growth control. Synechocystis mutants with mutations in $s s a A$ show delayed recovery from the chlorotic state [Heilmann et al., 2017]. The authors of that work also performed in vivo pull-down analysis of the RNA polymerase complex, and the data suggest that the 6S RNA promotes the recruitment of the housekeeping sigma factor SigA to the RNA polymerase core complex, concurrently supporting displacement of group 2 sigma factors, such as SigB, C and E. An early transition back to SigA-dependent transcription explains the extensive transcriptional response observed at the onset of resuscitation in the study by Klotz et al. [2016].

\section{The Second Phase of Resuscitation: Restoration of}

Photosynthesis

Only after the re-launch of the basic components of the metabolic machinery can the cells afford to re-install the photosynthetic machinery. This is because it imposes a high metabolic burden on the cells through the coordinated synthesis of lipids for thylakoid membranes, the large amounts of tetrapyrroles and all the proteins required for the photosynthetic complexes, from the PBS antenna to the reaction centres. The timing of chlorophyll biosynthesis is especially crucial, because free chlorophyll is toxic to the cells. All these processes must, therefore, occur in a highly coordinated manner. How this is achieved is still poorly understood. The two aforementioned transcriptome and proteome studies [Klotz et al., 2016; Spat et al., 2018], however, provide preliminary in-

Resuscitation from Nitrogen

Starvation-Induced Dormancy sights into this process. Remarkably, the first cellular response upon nitrogen addition is in fact to suppress any residual photosynthetic activity. This early negative impact on photosynthesis is in good agreement with the observation that the function of some of the early regulated gene products is to prevent premature synthesis of photosynthetic components. For example, transient expression of the non-coding RNA PsrR1 supresses chlorophyll biosynthesis and PBS gene expression [Georg et al., 2014] and expression of several PSII-related and chlorophyll biosynthesis-related genes are repressed in the early stage of resuscitation. The logic of this strategy might also be related to the diurnal lifestyle of cyanobacteria. In nature, the cyanobacteria are constantly subjected to the daily availability of light energy for a certain length of time. The awakening of the cells relies exclusively on glycogen-dependent energy metabolism, which allows utilization of nitrogen sources independent of light availability. This ensures a secure progression of resuscitation until the entire basic operation system is completely re-installed. Only then do the cells start to reconstitute the photosynthetic apparatus. The corresponding process works extremely precisely and is finely tuned at all levels. This is clearly illustrated for chlorophyll biosynthesis [Spat et al., 2018], whereby a decisive step in chlorophyll biosynthesis is the conversion of protochlorophyllide to chlorophyllide, for which light-dependent and light-independent isoenzymes exist in Synechocystis. At the proteome level, the enzymes are undetectable in the chlorotic state and in the early phase of resuscitation. The light-dependent protochlorophyllide reductase (Por) only becomes detectable in the middle phase of resuscitation [Spat et al., 2018], whereas the light-independent isoenzymes ChlB and ChlL are still absent. In agreement with this, the expression of the corresponding genes is repressed during the early phase of resuscitation (see above). This strategy ensures that chlorophyll biosynthesis can start as soon as the basic operation system is re-installed and light is available. The final biosynthetic enzyme, chlorophyll synthase (ChlG) is already present prior to the induction of Por synthesis, so that using this strategy the cells are prepared to convert the highly toxic chlorophyllide molecule into chlorophyll $a$, which is then incorporated into chlorophyll-binding proteins, which have to be synthesized simultaneously.

Another crucial step in the re-installation of the photosynthetic machinery is the assembly of the photosynthetic protein complexes in newly synthesized thylakoid membranes. Here, quantitative analyses of the phosphoproteome have yielded first intriguing insights into what 
is occurring. Among the phosphoproteins whose degree of phosphorylation changed dynamically over the time course of resuscitation, the largest functional group is indeed the proteins related to photosynthesis [Spat et al., 2018]. These proteins can be grouped in 5 classes according to the pattern of phosphorylation change. The few residual proteins of the PBS rods are highly phosphorylated during chlorosis and the phosphorylation level gradually decreases during resuscitation. Phosphorylation of the phycobiliproteins could be related to protein stability and turnover. Other photosynthetic proteins, such as PBS core proteins or some components of PSI and PSII, show a transient increase in phosphorylation. The Vipp1 proteins, for example, which play a decisive role in thylakoid membrane biogenesis [Heidrich et al., 2017], show a particularly complex phosphorylation pattern with 8 detected phosphorylation sites, occurring on 2 clustered regions of the protein. The phosphorylation level remains high during the initial resuscitation phase, with some sites even showing initially increased phosphorylation, until time point $24 \mathrm{~h}$. Subsequently, phosphorylation of all sites decreases, which occurs concomitantly with the accumulation of newly synthesized Vipp1 protein and the emergence of stacked thylakoid membranes. Vipp1 was shown to form large multimeric structures that form rings and rods [Theis et al., 2019] and control the formation of thylakoid membranes [Westphal et al., 2001]. Phosphorylation of Vipp1 might thus keep this protein in an inactive state, until the progression of resuscitation advances to the stage where Vippl activity comes into play. Based on this study, protein phosphorylation has thus emerged to add another layer of regulator complexity controlling the timing of resuscitation.

\section{Conclusions}

The analysis of the nitrogen starvation-induced transition of Synechocystis into a dormant-like chlorotic state and resuscitation form this state has revealed new insights into dormancy and awakening. Organisms from most diverse origins tune down cellular activities to a minimum in order to survive extended periods of adverse environmental conditions, such as nutrient starvation. Under these circumstances, they all need to find the answer to a major issue: What is the minimal activity necessary to maintain viability?

In order to resuscitate cells from the dormant state, a minimal initial energy is necessary, and a minimal set of the most fundamental cellular functions has to be maintained; in order to make new protein, at least some ribosomes and translation factors need to be in place. This minimal set of cellular activities represents the organism's equivalent of the BIOS, the basic input/output system of a computer, that helps re-boot the operation system after being switched on. In analogy, the same tasks have to be performed when resuscitation from the dormant state is executed. We have learned by using chlorosis in Synechocystis as a model system that evolution has prepared cells with a sophisticated genetic program to achieve this task. Moreover, it appears that already at the stage when the cells enter the dormant state, they anticipate the future awakening and prepare essential components to allow rapid resuscitation. The revival of nutrient starvation-induced Bacillus subtilis spores is another example of the existence of a highly organized developmental program to awaken from dormancy and reconstruct a cell [Sinai et al., 2015]. Another lesson learned from these investigations is the identification of the functions of genes that have been annotated as "hypothetical," since their roles appear only under extreme conditions, and some of these may even belong to the cellular BIOS.

\section{Conflict of Interest Statement}

The authors have no conflicts of interest to declare.

\section{Funding Sources}

Work in the Forchhammer laboratory was funded by the RTG 1708 "Molecular principles of bacterial survival strategies" and by DFG-funded Research Unit SCyCode (FOR2816). We further acknowledge support by Open Access Publishing Fund of University of Tübingen and Infrastructural Funding from the Cluster of Excellence EXC 2124 “Controlling Microbes to Fight Infections.”

\section{Author Contributions}

Conceptualization: K.F.; writing, original draft preparation: K.F.; review and editing: N.N., S.D. and K.F.

\section{References}

Al Dahouk S, Jubier-Maurin V, Neubauer H, Köhler S. Quantitative analysis of the Brucella suis proteome reveals metabolic adaptation to long-term nutrient starvation. BMC Microbiol. 2013;13(1): 199 .

Allen MM, Smith AJ. Nitrogen chlorosis in bluegreen algae. Arch Mikrobiol. 1969;69(2):11420.

Arias CR, LaFrentz S, Cai W, Olivares-Fuster O. Adaptive response to starvation in the fish pathogen Flavobacterium columnare: cell viability and ultrastructural changes. BMC Microbiol. 2012;12(1):266.
86

Microb Physiol 2021;31:78-87 DOI: $10.1159 / 000515742$
Neumann/Doello/Forchhammer 
Asayama M, Imamura S, Yoshihara S, Miyazaki A, Yoshida N, Sazuka T, et al. SigC, the group 2 sigma factor of RNA polymerase, contributes to the late-stage gene expression and nitrogen promoter recognition in the cyanobacterium Synechocystis sp. strain PCC 6803. Biosci Biotechnol Biochem. 2004;68(3):477-87.

Azuma M, Osanai T, Hirai MY, Tanaka K. A response regulator Rre37 and an RNA polymerase sigma factor SigE represent two parallel pathways to activate sugar catabolism in a cyanobacterium Synechocystis sp. PCC 6803. Plant Cell Physiol. 2011;52(2):404-12.

Baers LL, Breckels LM, Mills LA, Gatto L, Deery MJ, Stevens TJ, et al. Proteome mapping of a cyanobacterium reveals distinct compartment organization and cell-dispersed metabolism. Plant Physiol. 2019;181(4):1721-38.

Bolay P, Muro-Pastor MI, Florencio FJ, Klähn S. The distinctive regulation of cyanobacterial glutamine synthetase. Life (Basel). 2018;8(4).

Boresch K. Zur Physiologie der Blaualgenfarbstoffe. Lotos (Prag). 1910;58:344-345.

Collier JL, Grossman AR. A small polypeptide triggers complete degradation of light-harvesting phycobiliproteins in nutrient-deprived cyanobacteria. EMBO J. 1994;13(5):1039-47.

Damrow R, Maldener I, Zilliges Y. The multiple functions of common microbial carbon polymers, glycogen and PHB, during stress responses in the non-diazotrophic cyanobacterium Synechocystis sp. PCC 6803. Front Microbiol. 2016;7:966.

Doello S, Burkhardt M, Forchhammer K. The essential role of sodium bioenergetics and ATP homeostasis in the developmental transitions of a cyanobacterium. Curr Biol. 2021.

Doello S, Klotz A, Makowka A, Gutekunst K, Forchhammer K. A specific glycogen mobilization strategy enables rapid awakening of dormant cyanobacteria from chlorosis. Plant Physiol. 2018;177(2):594-603.

Georg J, Dienst D, Schürgers N, Wallner T, Kopp D, Stazic D, et al. The small regulatory RNA SyR1/ PsrR1 controls photosynthetic functions in cyanobacteria. Plant Cell. 2014;26(9):3661-79.

Görl M, Sauer J, Baier T, Forchhammer K. Nitrogen-starvation-induced chlorosis in Synechococcus PCC 7942: adaptation to longterm survival. Microbiology. 1998;144(9): 2449-58.

Grossman AR, Schaefer MR, Chiang GG, Collier JL. The phycobilisome, a light-harvesting complex responsive to environmental conditions. Microbiol Rev. 1993;57(3):725-49.

Gründel M, Scheunemann R, Lockau W, Zilliges Y. Impaired glycogen synthesis causes metabolic overflow reactions and affects stress responses in the cyanobacterium Synechocystis sp. PCC 6803. Microbiology. 2012;158(Pt 12): 3032-43.

Hauf W, Schlebusch M, Hüge J, Kopka J, Hagemann M, Forchhammer K. Metabolic changes in Synechocystis PCC6803 upon nitrogenstarvation: excess NADPH sustains polyhydroxybutyrate accumulation. Metabolites. 2013;3(1):101.
Heidrich J, Thurotte A, Schneider D. Specific interaction of IM30/Vipp1 with cyanobacterial and chloroplast membranes results in membrane remodeling and eventually in membrane fusion. Biochim Biophys Acta. 2017; 1859(4):537-49.

Heilmann B, Hakkila K, Georg J, Tyystjärvi T, Hess WR, Axmann IM, et al. 6S RNA plays a role in recovery from nitrogen depletion in Synechocystis sp. PCC 6803. BMC Microbiol. 2017a;17(1):229.

Hickman JW, Kotovic KM, Miller C, Warrener P, Kaiser B, Jurista T, et al. Glycogen synthesis is a required component of the nitrogen stress response in Synechococcus elongatus PCC 7942. Algal Res. 2013;2(2):98-106.

Imamura S, Asayama M. Sigma factors for cyanobacterial transcription. Gene Regul Syst Bio. 2009;3:65-87.

Keren I, Minami S, Rubin E, Lewis K. Characterization and transcriptome analysis of Mycobacterium tuberculosis persisters. mBio. 2011;2(3):e00100-11.

Klotz A, Georg J, Bučinská L, Watanabe S, Reimann V, Januszewski W, et al. Awakening of a dormant cyanobacterium from nitrogen chlorosis reveals a genetically determined program. Curr Biol. 2016;26(21):2862-72.

Klotz A, Reinhold E, Doello S, Forchhammer K. Nitrogen starvation acclimation in Synechococcus elongatus: redox-control and the role of nitrate reduction as an electron sink. Life (Basel). 2015;5(1):888.

Koch M, Berendzen KW, Forchhammer AK. On the role and production of polyhydroxybutyrate (PHB) in the cyanobacterium Synechocystis sp. PCC 6803. Life (Basel). 2020;10(4).

Koch M, Doello S, Gutekunst K, Forchhammer K. PHB is produced from glycogen turn-over during nitrogen starvation in Synechocystis sp. PCC 6803. Int J Mol Sci. 2019;20(8):1942.

Moreno-Gámez S, Kiviet DJ, Vulin C, Schlegel S, Schlegel K, van Doorn GS, et al. Wide lag time distributions break a trade-off between reproduction and survival in bacteria. Proc Natl Acad Sci USA. 2020;117(31):18729-36.

Osanai T, Oikawa A, Numata K, Kuwahara A, Iijima H, Doi Y, et al. Pathway-level acceleration of glycogen catabolism by a response regulator in the cyanobacterium Synechocystis species PCC 6803. Plant Physiol. 2014;164(4):1831-41.

Perez R, Wörmer L, Sass P, Maldener I. A highly asynchronous developmental program triggered during germination of dormant akinetes of filamentous diazotrophic cyanobacteria. FEMS Microbiol Ecol. 2018;94(1). https:// doi.org/10.1093/femsec/fix131.

Pils D, Schmetterer G. Characterization of three bioenergetically active respiratory terminal oxidases in the cyanobacterium Synechocystis sp. strain PCC 6803. FEMS Microbiol Lett. 2001;203(2):217-22.

Reyes JC, Florencio FJ. A mutant lacking the glutamine synthetase gene $(g \ln A)$ is impaired in the regulation of the nitrate assimilation system in the cyanobacterium Synechocystis sp. strain PCC 6803. J Bacteriol. 1994;176(24):7516-23.
Robertson BR, Tezuka N, Watanabe MM. Phylogenetic analyses of Synechococcus strains (cyanobacteria) using sequences of $16 \mathrm{~S}$ rDNA and part of the phycocyanin operon reveal multiple evolutionary lines and reflect phycobilin content. Int J Syst Evol Microbiol. 2001; 51(Pt 3):861-71.

Sauer J, Gorl M, Forchhammer K. Nitrogen starvation in Synechococcus PCC 7942: involvement of glutamine synthetase and NtcA in phycobiliprotein degradation and survival. Arch Microbiol. 1999;172(4):247-55.

Sauer J, Schreiber U, Schmid R, Völker K, Forchhammer K. Nitrogen starvation-induced chlorosis in Synechococcus PCC 7942. Lowlevel photosynthesis as a mechanism of longterm survival. Plant Physiol. 2001;126(1): 233-43.

Schlebusch M, Forchhammer K. Requirement of the nitrogen starvation-induced protein Sll0783 for polyhydroxybutyrate accumulation in Synechocystis sp. strain PCC 6803. Appl Environ Microbiol. 2010;76(18):61017.

Schwarz R, Forchhammer K. Acclimation of unicellular cyanobacteria to macronutrient deficiency: emergence of a complex network of cellular responses. Microbiology (Reading, Engl). 2005;151(Pt 8):2503-14.

Sinai L, Rosenberg A, Smith Y, Segev E, Ben-Yehuda $\mathrm{S}$. The molecular timeline of a reviving bacterial spore. Mol Cell. 2015;57(4):695707.

Spat P, Klotz A, Rexroth S, Macek B, Forchhammer K. Chlorosis as a developmental program in cyanobacteria: the proteomic fundament for survival and awakening. Mol Cell Proteomics. 2018.

Srivastava A, Summers ML, Sobotka R. Cyanobacterial sigma factors: current and future applications for biotechnological advances. Biotechnol Adv. 2020 May-Jun;40:107517.

Stephan DP, Ruppel HG, Pistorius EK. Interrelation between cyanophycin synthesis, L-arginine catabolism and photosynthesis in the cyanobacterium Synechocystis sp. strain PCC 6803. Z Naturforsch C J Biosci. 2000; 55(11囚12):927-42.

Theis J, Gupta TK, Klingler J, Wan W, Albert S, Keller S, et al. VIPP1 rods engulf membranes containing phosphatidylinositol phosphates. Sci Rep. 2019;9(1):8725.

Udikovic-Kolic N, Wichmann F, Broderick NA, Handelsman J. Bloom of resident antibioticresistant bacteria in soil following manure fertilization. Proc Natl Acad Sci USA. 2014; 111(42):15202-7.

Watzer B, Forchhammer K. Cyanophycin synthesis optimizes nitrogen utilization in the unicellular cyanobacterium Synechocystis sp. strain PCC 6803. Appl Environ Microbiol. 2018;84(20).

Westphal S, Heins L, Soll J, Vothknecht UC. Vipp1 deletion mutant of Synechocystis: a connection between bacterial phage shock and thylakoid biogenesis?. Proc Natl Acad Sci USA. 2001;98(7):4243-8. 